\title{
The impact of smoking on adherence to treatment for latent tuberculosis infection
}

\author{
Mélanie Lavigne ${ }^{1}$, Isabelle Rocher ${ }^{2}$, Colin Steensma ${ }^{1}$ and Paul Brassard*1
}

\author{
Address: ${ }^{1}$ McGill University Health Center - Division of Clinical Epidemiology - Royal Victoria Hospital, Montreal, Canada and ${ }^{2}$ McGill \\ University Health Center - Montreal Chest Institute, Montreal, Canada \\ Email: Mélanie Lavigne - mel_lavigne@ hotmail.com; Isabelle Rocher - isabelle.rocher@inspq.qc.ca; Colin Steensma - colin.steensma@phac- \\ aspc.gc.ca; Paul Brassard* - paul.brassard@mcgill.ca \\ * Corresponding author
}

Published: 14 March 2006

BMC Public Health2006, 6:66 doi:10.1 I86/147|-2458-6-66
Received: 30 September 2005

Accepted: 14 March 2006

This article is available from: http://www.biomedcentral.com/I47/-2458/6/66

(C) 2006Lavigne et al; licensee BioMed Central Ltd.

This is an Open Access article distributed under the terms of the Creative Commons Attribution License (http://creativecommons.org/licenses/by/2.0), which permits unrestricted use, distribution, and reproduction in any medium, provided the original work is properly cited.

\begin{abstract}
Background: Studies have shown an association between smoking and tuberculosis (TB) infection, disease and TB-related mortality. We hypothesized that smokers with latent tuberculosis infection (LTBI) are less likely to comply with their LTBI treatment regimen, thus increasing their risk of developing active disease. We thus documented the impact of smoking on adherence to LTBI treatment.

Method: Between 1998 and 2000, a convenience sample of patients undergoing treatment for LTBI completed a questionnaire on smoking status. Level of adherence to LTBI treatment was tested for associations with socio-demographic profile, and smoking status

Results: 320 patients were recruited, and $302(94 \%)$ completed the questionnaire. Smoking prevalence was $21 \% .72 \%$ of patients were adherent to LTBI treatment. Women (OR $=2.0 ; 95 \%$ Cl: I.2-3.3) and non-smokers (OR = I.8; 95\% Cl: I.0-3.3) were associated with adherence to LTBI treatment. Only gender was found as an independent predictor of adherence after adjusting for age and smoking status (OR $=1.9 ; 95 \% \mathrm{Cl}:$ 1.06-3.3).
\end{abstract}

Conclusion: Males and smokers need to have extra supervision to ensure compliance with LTBI treatment.

\section{Background}

Studies have shown an association between smoking and tuberculosis (TB) infection,[1] disease, [2-6] and TBrelated mortality. [7] Based on the premise that smokers tend to be less compliant to medication $[8,9]$ we hypothesized that smokers with latent tuberculosis infection (LTBI) are less likely to comply with their LTBI treatment regimen, thus increasing their risk of developing active disease. We thus set out to document the impact of smoking on adherence to LTBI.

\section{Methods}

For the 1998-2000 period, a convenience sample was taken of consecutive patients initiating treatment for LTBI who were given the opportunity to undergo a self-administered questionnaire (French-English) on baseline demographic characteristics. Patients were recruited at a specialized TB clinic located at the Montreal Chest Institute (MCI), which deals mainly but not exclusively with new adult immigrants and refugee claimants for whom immigration screening evaluation suggested inactive TB. [10] Eligibility of patients was defined according to their 
level of understanding of the languages of the survey. A written informed consent to participate in the study was obtained before administration of the questionnaire.

Smoking status and nicotine dependence was assessed by the nurse clinician. We used the Canadian tobacco use monitoring survey (CTUMS) terminology to define current and non- and ex-smokers. [11] Nicotine dependence in smokers was assessed using a 10 point visual scale, with 1 denoting minimum dependence and 10 denoting maximum, using a modified Fagerström test for nicotine dependence. [12] Subjects were classified as nicotine dependent if the score was 6 or higher.

The medical evaluation followed the Canadian Thoracic Society Guidelines for tuberculosis control [13] and included sputum smear and cultures when indicated. Adherence to therapy was assessed by the clinic nurses through pill counts, patient self-report and general attendance at the scheduled medical visits. Adherence was considered adequate if patients took more than $80 \%$ of total prescribed doses for the LTBI treatment regimen, with the remainder being considered non adherent.

Level of adherence to LTBI treatment was tested for associations with socio-demographic profile (age, gender, region of birth), and smoking status (current/non- and exsmokers). Univariate analysis using chi-square test, Fisher's exact test or t-test was used to compare various characteristics of interest to LTBI treatment adherence. Significance was defined at the $\mathrm{p}<=0.05$ level. We performed a multiple logistic regression analysis using a stepwise approach to estimate the independent contribution of significant characteristics found in the univariate analysis towards adherence to LTBI. Data analysis was done using the SAS program (version 8.2). Ethical approval was obtained from the Montreal Chest Institute ethics committee.

\section{Results}

337 patients were initially asked to participate and 320 were recruited (180 males) with a mean age of $35.5(\mathrm{SD} \pm$ 10.8 ) years old. We obtained adherence status in 302/320 (94\%) participants. More than $87 \%$ (281/320) of our study group was found to be foreign born and $64 \%$ of the latter had immigrated to Canada within the previous 5 years. Most of our participants (33\%) came from the Indian sub continent (mostly India and Pakistan) and $17 \%$ from North Africa (mostly Algeria and Morocco). Smoking prevalence was $21 \%$ (28\% and $20 \%$ among Canadian born and foreign born respectively). The overall mean nicotine dependence among smokers was low (2.9/ $10(S D \pm 2.4)$; median 3.0) and reflected the fact that only $17 \%$ of current smokers reported being tobacco dependent (Table). Smokers smoked in general less than 12 ciga- rettes per day (48\%), while $23 \%$ smoked at least 25 cigarettes a day (the equivalent of a half pack and a full pack of cigarettes respectively). Overall, adequate adherence to LTBI treatment was found in 217/302 (72\%) participants. In the univariate analysis, factors associated with adherence to LTBI treatment included being female (odds ratio $(\mathrm{OR})=2.0$; 95\% confidence interval $(\mathrm{CI}): 1.2-$ 3.3 ) and being a non-smoker ( $\mathrm{OR}=1.8$; 95\% CI: 1.0 3.3). Characteristics such as age, region of birth, and nicotine dependence among smokers were not associated with adherence to LTBI (Table). In the multiple logistic regression analysis, only being female $(\mathrm{OR}=1.9 ; 95 \% \mathrm{CI}$ : 1.1-3.3) was independently associated with adherence when adjusted for age and smoking status.

\section{Discussion}

In this study, smoking prevalence was found among $21 \%$ of our study population and was more prevalent in men $(33 \%)$ than in women $(6 \%)$. Our finding is comparable to the prevalence found in the respective region of birth of the participants. [14] As $88 \%$ of our study group were from the developing world, it was not surprising to see that men were more likely to smoke than woman. $[15,16]$

Adequate adherence to LTBI was found in $72 \%$ of patients and is consistent with previous studies done at the MCI. [10] In our univariate analysis, men and smokers were less likely to be compliant to treatment. The multiple logistic regression analysis showed that gender was the only independent predictor of adherence. Males may be less compliant to LTBI treatment for economic reasons. In various cultures, men are the main contributors to the family income and cannot afford to take time out for a medical visit to a clinic. Indeed, travel time to and from the clinic represents time absent from work and potentially less money earned. [17] This speculation is supported by the finding among our study group that men were less likely than women to report attending regular medical visits ( $\mathrm{p}$ $<0.001$ ). Another plausible reason would be that males are less likely to adhere to healthy behaviours and prevention messages such as smoking cessation and adherence to treatment.

Certain limitations in this study have to be recognized. The self-report of smoking status could have been biased by a certain degree of social desirability from the respondent which may have underestimated the true prevalence of smoking. Characteristics such as economic status and language barriers/comprehension (although the latter was initially addressed in the participant selection) might have also influenced adherence, especially in the foreign-born, but were not sought after in this study. Our convenience sample of participants was nonetheless representative of the overall MCI clinic attendance with respect to basic socio-demographic factors, $[18,19]$ although generaliza- 
Table I: Patient characteristics associated with LTBI treatment adherence.

\begin{tabular}{|c|c|c|c|}
\hline \multirow[t]{2}{*}{ Characteristics } & \multicolumn{3}{|c|}{ Adherence } \\
\hline & Non adherent $\mathbf{N}=\mathbf{8 5}(\%)$ & Adequate $\mathrm{N}=217(\%)$ & p-value \\
\hline \multicolumn{4}{|l|}{ Gender } \\
\hline Male & $58(68)$ & $113(52)$ & 0.01 \\
\hline Female & $27(32)$ & $104(48)$ & \\
\hline \multicolumn{4}{|l|}{ Age in years } \\
\hline $15-30$ & $35(4 I)$ & $78(36)$ & \\
\hline $31-45$ & $39(46)$ & $97(45)$ & 0.4 \\
\hline$>45$ & $11(13)$ & $42(19)$ & \\
\hline Mean age ( \pm S.D.) & $35( \pm 10)$ & $36( \pm 11)$ & 0.8 \\
\hline \multicolumn{4}{|l|}{ Region of origin } \\
\hline Canadian born & $11(13)$ & $26(12)$ & 0.8 \\
\hline Foreign born & $74(87)$ & $191(88)$ & \\
\hline Africa & $21(25)$ & $58(27)$ & \\
\hline Asia & $33(39)$ & $85(39)$ & \\
\hline Europe & $4(5)$ & $16(8)$ & \\
\hline Latin America & $16(18)$ & $30(14)$ & \\
\hline \multicolumn{4}{|l|}{ Smoking status } \\
\hline Current smoker & $25(29)$ & $40(18)$ & 0.04 \\
\hline Non/ex-smoker & $60(7 I)$ & $177(82)$ & \\
\hline \multicolumn{4}{|l|}{ Nicotine dependence ${ }^{*} \dagger$} \\
\hline$<6$ & $10(77)$ & $15(88)$ & 0.6 \\
\hline$\geq 6$ & $3(23)$ & $2(12)$ & \\
\hline
\end{tabular}

*: Modified Fagerström test scores among smokers only $\dagger \mathrm{n}=30$ SD: Standard deviation

bility of our findings to other clinical sites is somewhat limited. We also had a small proportion (5\%) of eligible subjects who refused to participate. We were not able to characterize these individuals but there is no reason to think that they were any different from our study group regarding the variables of interest.

\section{Conclusion}

Overall, our findings indicate that males and smokers need to have extra supervision to ensure compliance with LTBI treatment.

\section{Abbreviations}

TB-tuberculosis

\section{LTBI-latent tuberculosis infection}

MCI-Montreal Chest Institute

CTUMS-Canadian tobacco use monitoring survey

OR-odds ratio

CI-confidence interval

SD-standard deviation

\section{Competing interests}

The author(s) declare that they have no competing interests.

\section{Authors' contributions}

ML participated in the design, collected and updated data, performed part of the statistical analysis and drafted the manuscript. IR conceived the study, and participated in its design and helped to draft the manuscript. CS performed part of the statistical analysis and helped to draft the manuscript. PB supervised and insured coordination of the study and helped to draft the manuscript. All authors read and approved the final manuscript.

\section{Acknowledgements}

We would like to acknowledge the invaluable collaboration of the tuberculosis clinic nursing team and the medical archives of the Montreal Chest Institute. Ms. Lavigne was supported by a Summer Student Scholarship from McGill University. Dr Brassard is supported by a New Investigator Career Award from the Canadian Institutes of Health Research (CIHR).

\section{References}

I. den Boon S, van Lill SW, Borgdorff MW, Verver S, Bateman ED, Lombard CJ, Enarson DA, Beyers N: Association between smoking and tuberculosis infection: a population survey in a high tuberculosis incidence area. Thorax 2005, 60(7):555-7.

2. Lienhardt C, Fielding K, Sillah JS, Bah B, Gustafson P, Warndorff D, Palayew M, Lisse I, Donkor S, Diallo S, Manneh K, Adegbola R, Aaby $\mathrm{P}$, Bah-Sow O, Bennett S, McAdam K: Investigation of the risk factors for tuberculosis: a case-control study in three countries in West Africa. Int J Epidemiol 2005, 34(4):9|4-23. 
3. Maurya V, Vijayan VK, Shah A: Smoking and tuberculosis: an association overlooked. Int J Tuberc Lung Dis 2002, 6(I I):942-5I.

4. Kolappan C, Gopi PG: Tobacco smoking and pulmonary tuberculosis. Thorax 2002, 57( I I):964-6.

5. Leung CC, Yew WW, Chan CK, Tam CM, Lan CW, Chang KC, Chau $\mathrm{CH}$, Lau KS, Law WS: Smoking and tuberculosis in Hong Kong. Int J Tuberc Lung Dis 2003, 7(10):980-6.

6. Yach D: Partnering for better lung health: improving tobacco and tuberculosis control. Int J Tuberc Lung Dis 2000, 4(8):693-7.

7. Gajalakshmi V, Peto R, Kanaka TS, Jha P: Smoking and mortality from tuberculosis and other diseases in India: retrospective study of $\mathbf{4 3 0 0 0}$ adult male deaths and $\mathbf{3 5 0 0 0}$ controls. Lancet 2003, 362(9383):507-15.

8. McNagny SE, Ahluwalia JS, Clark WS, Resnicow KA: Cigarette smoking and severe uncontrolled hypertension in inner-city African Americans. Am J Med 1997, I03(2): 121-7.

9. Shea S, Misra D, Ehrlich MH, Field L, Francis CK: Correlates of nonadherence to hypertension treatment in an inner-city minority population. Am J Public Health 1992, 82(12):1607-12.

10. Dasgupta K, Schwartzman K, Marchand R, Tennenbaum TN, Brassard $P$, Menzies D.: Comparison of cost-effectiveness of tuberculosis screening of close contacts and foreign-born populations. Am J Respir Crit Care Med 2000, I 62(6):2079-86.

II. Canadian Tobacco Use Monitoring Survey (CTUMS) [http:/ /www.hc-sc.gc.ca/hl-vs/tobac-tabac/research-recherche/stat/ctumsesutc/terminolog/index e.html]. (Last viewed: on 21/09/2005)

12. Heatherton TF, Kozlowski LT, Frecker RC, Fagerstrom KO: The Fagerstrom Test for Nicotine Dependence: a revision of the Fagerstrom Tolerance Questionnaire. $\mathrm{Br} J$ Addict 1991, 86(9): I II $19-27$.

13. Canadian Lung Association: Canadian Tuberculosis Standards 5th edition. Edited by: Long R. Ottawa, Health Canada; 2000.

14. World Health Organization: Tobacco Free Initiative(TFI). The Tobacco Atlas 2004 [http://www.who.int/tobacco/statistics/ tobacco atlas/en/]. (Last viewed on January 92006 )

15. Rogers RG, Crank J: Ethnic differences in smoking patterns: finding from NHIS. Public Health Rep 1998, 103(4):387-93.

16. WHO 's Tobacco or Health: A Global Youth Tobacco Report 1997.

17. O'Boyle SJ, Power J, Ibrahim MY, Watson JP: Factors affecting patient compliance with anti-tuberculosis chemotherapy using the directly observed treatment, short-course strategy (DOTS). Int J Tuberc Lung Dis 2002, 6(4):307-I2.

18. Yee D, Valiquette C, Pelletier M, Parisien I, Rocher I, Menzies D: Incidence of serious side effects from first-line antituberculosis drugs among patients treated for active tuberculosis. $\mathrm{Am} \mathrm{J}$ Respir Crit Care Med 2003, I67( I I):1472-7.

19. Richards B, Kozak R, Brassard P, Menzies D, Schwartzman K: Tuberculosis surveillance among new immigrants in Montreal. Int J Tuberc Lung Dis 2005, 9(8):858-64.

\section{Pre-publication history}

The pre-publication history for this paper can be accessed here:

http://www.biomedcentral.com/1471-2458/6/66/prepub

\section{Publish with Biomed Central and every scientist can read your work free of charge}

"BioMed Central will be the most significant development for disseminating the results of biomedical research in our lifetime. "

Sir Paul Nurse, Cancer Research UK

Your research papers will be:

- available free of charge to the entire biomedical community

- peer reviewed and published immediately upon acceptance

- cited in PubMed and archived on PubMed Central

- yours - you keep the copyright
BioMedcentral 